\title{
A Rare Case of Dyspnoea the Parsonage-Turner Syndrome
}

\author{
Maurizio Marvisi ${ }^{\mathrm{a}, \mathrm{c}}$, Laura Balzarini ${ }^{\mathrm{a}}$, Chiara Mancini ${ }^{\mathrm{a}}$, \\ Massimo Confortini ${ }^{a}$, Enrico Betri ${ }^{b}$
}

\begin{abstract}
Amyotrophic neuralgia is an inflammatory and idiopathic neuropathy characterised by neuropathic pain. It was first described in 1948 as an affectation of just the brachial plexus and was called Parsonage-Turner syndrome. Although this syndrome is more frequent in the brachial plexus, it may affect the phrenic nerve concomitantly or in isolation, a diagnosis that is particularly difficult without strong clinical evidence.We describe the case of a young male presenting a monolateral phrenic palsy two years after a classic brachial neuropathy. The patient showed diffuse skin lypomas and achieved complete clinical remission in 6 months.
\end{abstract}

Keywords: Parsonage-turner; Phrenic nerve palsy; Lypoma

\section{Introduction}

Unilateral diaphragmatic paralysis may be idiopathic or secondary to multiple causes, the most common being a tumour with phrenic nerve involvement or surgical trauma. In its idiopathic form, viral infection has been identified as the most likely aetiology and in some cases a genetic mechanism may be involved. In highly symptomatic patients we should suspect involvement of the controlateral diaphragm or of the intercostal muscles. Neuralgic amyotrophy (NA), also called Parsonage-Turner syndrome (PST), is an idiopathic neuropathy characterized by the acute onset of neuropathic pain followed by weakness and atrophy of the involved musculature;

\footnotetext{
Manuscript accepted for publication January 27, 2012

${ }^{\mathrm{a}}$ Department of Internal Medicine and Pneumology, Clinica Figlie di S, Camillo, Italy

${ }^{\mathrm{b}}$ Department of Histopathology, Cremona, Italy

${ }^{\mathrm{c} C}$ Corresponding author: Maurizio Marvisi. Email: mmarvis@alice.it
}

doi:10.4021/jmc551w in rare cases a phrenic nerve involvement may be seen $[1,2]$.

\section{Case Report}

A 30-year-old man presented at our outpatient service with a two-week history of fatigue, exertional dyspnoea and orthopnoea. He worked as a journalist in local TV. His family history was silent. The patient's medical history included an episode, two years before, of sudden onset of right shoulder pain followed by weakness, dysesthaesia and numbness in the right arm. The diagnosis was NA. He was treated with NSAIDS, neuroleptics and transcutaneous electrical nerve stimulation (TENS). A complete clinical recovery was gained after 12 weeks.

His ongoing medications included short acting $\beta 2$, used as needed for a mild asthma.

\section{Physical examination and laboratory findings}

Physical examination revealed a normal cardiac rhythm and a normal respiratory rate at rest. Oxygen saturation was 97\% and blood pressure was normal. Chest percussion and auscultation suggested paralysis of the right hemidiaphragm. We showed the presence of painless, firm, mobile subcutaneous nodules without overlying epidermal involvement with a predilection for trunk and extremities. Laboratory evaluation revealed a mild eosinophilia.

Standard chest radiography and sniff test confirmed the right hemidiaphragm paralysis.

Pulmonary function tests performed in upright and supine position showed consistent data: Vital capacity (VC) upright $70 \%$ of predicted value, $\mathrm{VC}$ in supine position $54 \%$; FEV1 upright $64 \%$ and $45 \%$ supine. Magnetic resonance imaging (NMR) of the cervical spine and a chest CT scan were normal. We performed a skin biopsy on a nodule located in the right arm; the histological diagnosis was of lypoma (Fig. 1).

The definitive diagnosis was PST disease with unilateral diaphragm paralysis due to phrenic nerve involvement and subcutaneous lypomas. The patient achieved a complete clinical resolution after 6 months. 


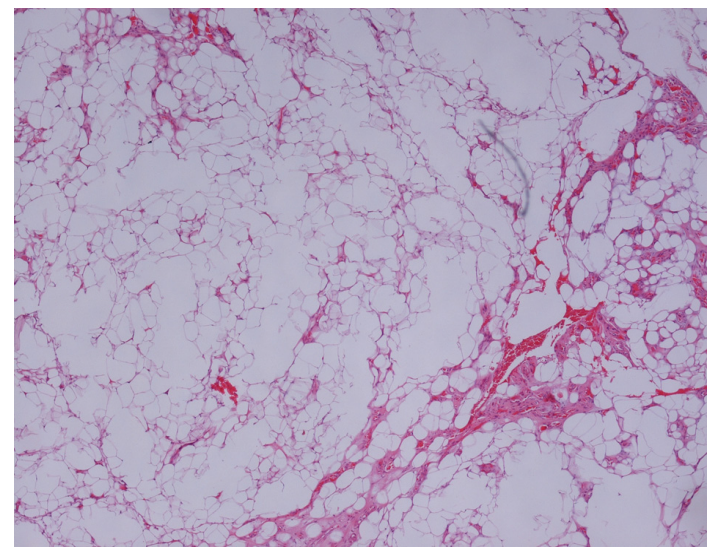

Figure 1. Hematoxylin-Eosin $\times 5$. Normal adipocytes interspersed with streaks of collagen fibers with poor cellular component.

\section{Discussion}

Brachial plexitis was first described as a distinct clinical entity in 1943 by Spillane, and before that, in 1897 Feinburg described similar clinical presentations. In 1948, in a large study Parsonage and

Turner described 136 cases of neurologic amyotrophy, called "shoulder-girdle syndrome".

PST syndrome is a rare disorder affecting the upper trunk of the brachial plexus or one of the shoulder girdle's peripheral nerves. In the literature rare cases are described with involvement of the middle and lower trunks [1]. The annual incidence is 2 to 3 per 100,000 persons per year. The disorder has a peak incidence between the third and fifth decades and is male predominant, 2-4:1. NA can occur as a sporadic disorder (idiopathic) or as an autosomal dominant hereditary trait, known as hereditary NA (HNA), which predisposes to recurrent attacks of peripheral neuropathy $[2,3]$.

The patients declare abrupt onset and severe shoulder pain, always self-limiting, lasting from 1 to 2 weeks, in rare cases longer periods, and progressive motor weakness [2].

The acute brachial plexus may be confused with an isolated peripheral nerve lesion, suggesting local compression, while the pathophysiology is probably a diffuse proximal inflammation.

Similar symptoms are presented by cervical radiculopathy, shoulder/upper extremity pathology (rotator cuff tendonopathy, subacromial bursitis etc.), peripheral-nerve compression, tumour, acute poliomyelitis, amyotrophic lateral sclerosis, thoracic outlet syndrome, postviral syndrome and brachial plexopathy.

The diagnosis becomes more recognizable when the pain decreases spontaneously and weakness develops. The etiology of the disease is unknown, with $25 \%$ of cases occurring after viral infection and $15 \%$ after immunization $[1,2]$.

There are some reports of Parsonage-Turner syndrome associated with rheumatologic diseases such as systemic lupus erythematosus and polyarteritis nodosa [4]. The precise location (axon, dendrites or neuromuscular junction) of neuronal injury and etiology of this neuropathy are unknown; however, autoimmune injury leading to focal demyelination or axonal degeneration has been suggested as a pathogenic mechanism. Treatment of nonphrenic NA is mainly supportive, including analgesics, physiotherapy and reassurance. Corticosteroids have been administered to reduce pain, but they have not been shown to influence the course of the illness. Anecdotal reports of success with plasma exchange and IV Ig have been recorded.

Recovery is sporadic, and some patients are left with pain and/or paresis after 3 years. Full recovery of sensory deficits is seen in one third of patients [5].

In the English language literature there are sporadic cases of respiratory system involvement, above all phrenic nerve palsies that in rare cases may even be bilateral [5-8]. Rarer reports describe laryngeal and bilateral vocal cord paresis due to involvement of both recurrent laryngeal nerves [6]. The incidence of extrabrachial involvement (including the phrenic nerve) is higher (56\%) in the hereditary subtype than in the idiopathic one (17\%) [9]. PST causes more than half of cases with isolated idiopathic phrenic nerve paresis.

Phrenic nerve involvement can also be suggested by lung function tests that show restriction and

decreased inspiratory pressures with preserved functional residual capacity. Expiratory reserve volume (ERV) and maximum expiratory mouth pressure are generally preserved in isolated phrenic palsy. In patients with isolated phrenic nerve involvement due to NA, obtaining an accurate history of preceding pain, weakness and other sensory symptoms will lead to a correct diagnosis and avoid unnecessary investigations [5].

In patients with associated or isolated phrenic nerve involvement, the clinical outcome is variable.

Some degree of recovery is usual but may be slow or incomplete. Recovery may not commence for up to 3 years after diagnosis. The delay may be due to the long length of time over which the phrenic nerve must regenerate. In patients with severe dyspnoea, plication of the diaphragm may result in improved ventilation and gas exchange. It is suggested that plication be delayed for at least 2 years after diagnosis because delayed spontaneous recovery may be seen [5-8].

The novelty of our report resides in the long interval between the first episode of brachial NA and the phrenic nerve palsy (two years) and in the presence of diffuse skin involvement shown by diffuse subcutaneous lypomas $[2,3]$.

The clinical presentation is suggestive of HNA, an autosomal dominant disorder the associated findings of which include relative hypotelorism, occasional cleft palate, and skin folds or creases on the neck and forearm [3]. To our knowledge this is the first report describing skin lypomas 
probably associated with HNA syndrome. In recent years, a gene was localized to human chromosome $17 \mathrm{q} 25.3$, and some point mutation in the alternatively spliced SEPT9 gene encoding septin 9 proteins. These proteins that participate in cytokinesis and cellular trafficking have been widely studied for their relationship to benign and malignant neoplasia [3].

\section{Conclusions}

We describe the case of a young man who developed dyspnoea due to monolateral phrenic nerve paresis two years after an acute episode of NA involving the right arm. The patient showed diffuse cutaneous lypomas with predilection of trunks and extremities. He achieved a compete recovery after 6 months. PTS is an idiopathic neuropathy involving above all the brachial plexus, characterized by the acute onset of neuropathic pain followed by weakness and atrophy of the involved musculature. In rare cases the syndrome involves the phrenic nerve inducing monolateral or bilateral diaphragmatic palsy. The hereditary form may induce cutaneous alterations such as lypomas.

\section{References}

1. Feinberg JH, Radecki J. Parsonage-turner syndrome. HSS journal : the musculoskeletal journal of Hospital for Special Surgery. 2010;6(2):199-205.

2. van Alfen N, van Engelen BG. The clinical spectrum of neuralgic amyotrophy in 246 cases. Brain : a journal of neurology. 2006;129(Pt 2):438-450.
3. Hannibal MC, Ruzzo EK, Miller LR, Betz B, Buchan JG, Knutzen DM, Barnett K, et al. SEPT9 gene sequencing analysis reveals recurrent mutations in hereditary neuralgic amyotrophy. Neurology. 2009;72(20):17551759.

4. Allan SG, Towla HM, Smith CC, Downie AW, Clark JC. Painful brachial plexopathy: an unusual presentation of polyarteritis nodosa. Postgraduate medical journal. 1982;58(679):311-313.

5. Kalluri M, Huggins JT, Strange C. A 56-year-old woman with arm pain, dyspnea, and an elevated diaphragm. Chest. 2008;133(1):296-299.

6. Holtbernd F, Zehnhoff-Dinnesen AA, Duning T, Kemmling A, Ringelstein EB. An unusual case of neuralgic amyotrophy presenting with bilateral phrenic nerve and vocal cord paresis. Case reports in neurology. 2011;3(1):69-74.

7. Ikegami G, Abe T, Akasaka K, Kouyama A, Souma R, Matsuo T, Kouyama K, et al. Bilateral phrenic nerve paralysis manifested by orthopnea for 6 months in a patient with neuralgic amyotrophy. Internal medicine. 2009;48(24):2123-2127.

8. Blanco-Aparicio M, Montero-Martinez C, Couto-Fernandez D, Pernas B, Fernandez-Marrube M, Verea-Hernando $\mathrm{H}$. [Unilateral painful diaphragm paralysis as the only sign of amyotrophic neuralgia]. Archivos de bronconeumologia. 2010;46(7):390-392.

9. Hashizume H, Nishida K, Nanba Y, Shigeyama Y, Inoue $\mathrm{H}$, Morito Y. Non-traumatic paralysis of the posterior interosseous nerve. The Journal of bone and joint surgery. British volume. 1996;78(5):771-776. 